\title{
SYSTEMATIC REVIEW
}

\section{Three-dimensional imaging of soft and hard facial tissues in patients with craniofacial syndromes: a systematic review of methodological quality}

\author{
Arianne Lewyllie, Maria Cadenas De Llano-Pérula, Anna Verdonck and Guy Willems \\ Department of Oral Health Sciences - Orthodontics, KU Leuven and Dentistry, University Hospitals Leuven, Leuven, Belgium
}

\begin{abstract}
Objectives: To systematically review the methodological quality of three-dimensional imaging studies of patients with craniofacial syndromes and to propose recommendations for future research.

Methods: PubMed, Embase and Cochrane databases as well as Grey literature were electronically searched. Inclusion criteria were patients with genetic syndromes with craniofacial manifestations and three-dimensional imaging of facial soft and/or hard tissues. Exclusion criteria consisted of non-syndromic conditions or conditions owing to environmental causes, injury or trauma, facial soft and hard tissues not included in the image analysis, case reports, reviews, opinion articles. No restrictions were made for patients' ethnicity nor age, publication language or publication date. Study quality was evaluated using the Methodological Index for Non-Randomized Studies (MINORS).

Results: The search yielded 2228 citations of which 116 were assessed in detail and 60 were eventually included in this review. Studies showed a large heterogeneity in study design, sample size and patient age. An increase was observed in the amount of studies with time, and the imaging method most often used was CT. The most studied craniofacial syndromes were Treacher Collins, Crouzon and Apert syndrome. The articles could be divided into three main groups: diagnostic studies (34/60, 57\%), evaluation of surgical outcomes $(21 / 60,35 \%)$ and evaluation of imaging techniques $(5 / 60,8 \%)$. For comparative studies, the median MINORS score was $13(12-15,25-75$ th percentile), and for non-comparative studies, the median MINORS score was 8 (7-9, 25-75th percentile).

Conclusions: The median MINORS scores were only 50 and 54\% of the maximum scores and there was a lack of prospective, controlled trials with sufficiently large study groups. To improve the quality of future studies in this domain and given the low incidence of craniofacial syndromes, more prospective multicentre controlled trials should be set up.

Dentomaxillofacial Radiology (2017) 46, 20170154. doi: 10.1259/dmfr.20170154

Cite this article as: Lewyllie A, Cadenas De Llano-Pérula M, Verdonck A, Willems G. Three-dimensional imaging of soft and hard facial tissues in patients with craniofacial syndromes: a systematic review of methodological quality. Dentomaxillofac Radiol 2017; 46: 20170154.
\end{abstract}

Keywords: review; craniofacial abnormalities; imaging, three-dimensional; face; radiology

\section{Introduction}

Craniofacial syndromes can be considered as a collective term for a wide group of congenital, genetic disorders with multiple anomalies and major symptoms in the craniofacial area. All of these syndromes are relatively

Correspondence to: Dr Guy Willems, E-mail: guy.willems@kuleuven.be Received 21 April 2017; revised 12 November 2017; accepted 20 November 2017 rare with only few live births per year. Some of the most studied craniofacial syndromes are those associated with early closure of the cranial sutures (Pfeiffer, Apert, Crouzon syndrome) together with Treacher Collins and Goldenhar syndrome.

There is a wide variety in the severity of the craniofacial manifestations between the syndromes, ranging 
from a clear deformity of the entire craniofacial skeleton to much more subtle asymmetries. To determine the extensiveness of the deformity, medical imaging is often performed for diagnosis or in relation to surgical interventions. Originally, two-dimensional (2D) images and measurements were made. However, a face is intrinsically a three-dimensional (3D) object. Preservation of this nature has been made possible since the introduction of $3 \mathrm{D}$ imaging methods, which are now widely and frequently used to give a detailed representation of the craniofacial structures. ${ }^{1}$ However, some imaging procedures like CT or cone beam CT (CBCT) make use of ionizing radiation and their potential to harm developing tissues should be taken into account, especially in young children. In contrast, non-invasive imaging methods like MRI and 3D ultrasonography are also available and have their specific indications. ${ }^{2}$ Another non-invasive 3D modality is facial surface scanning, which provides imaging of the external facial soft tissues. This technique has been increasingly used to investigate the relation between facial morphology and the underlying genes, the so-called "phenotype-genotype interaction".

The primary aim of this review was to evaluate the methodological quality of 3D imaging studies of subjects with different craniofacial syndromes. The secondary aim was to make recommendations for performing future studies. We included a broad range of syndromes to gather as much information as possible on 3D imaging. We additionally evaluated the following:

(1) Which 3D imaging methods were used?

(2) What were the indications for taking these images?

(3) What was the evolution over time of the study design and type of imaging methods?

\section{Methods and materials}

The protocol for this review followed a review structure developed prior to the literature search. Reporting follows the Preferred Reporting Items for Systematic Reviews and Meta-Analyses guidelines ${ }^{3}$ and the Population, Intervention, Comparison and Outcome of Interest process. ${ }^{4}$

\section{Eligibility criteria}

P. Patients with craniofacial syndromes

I. 3D imaging

C. 2D imaging technique, no comparison or other comparison group (non-affected side or healthy controls)

O. Assessment of facial soft and/or hard tissues

Publications using 3D imaging techniques for assessing facial soft and hard tissues in patients with genetic syndromes with craniofacial manifestations were eligible for inclusion. Exclusion criteria were: articles with a study group consisting only of patients with non-syndromic conditions or conditions owing to environmental causes (like non-syndromic cleft palate or obstructive sleep apnoea syndrome (OSAS), injury or trauma, facial soft and hard tissues not included in the image analysis, case reports, reviews, opinion articles. No restriction was made for patients' ethnicity nor age because in patients with craniofacial syndromes images are often taken prior to surgical interventions, and these interventions are commonly performed in early childhood. Also, no restriction was made for publication language nor publication date.

\section{Information sources}

Until the 15th of April 2017, literature was electronically searched using the PubMed, EMBASE and Cochrane databases. In addition, reviews on the subject and bibliographies of articles included in this review were manually checked for references (further referred to as 'other sources'). Grey literature was also searched through the GreyLit database (www.greylit.org).

\section{Search and study selection}

A search strategy was developed with the help of an experienced librarian specialized in health sciences and affiliated with the biomedical library of the KU Leuven, Belgium. The search strings used for PubMed, Embase and Cochrane databases are shown in Table 1.

After the removal of duplicate records, the initial study selection was based on titles and abstracts. Then, the full text was retrieved for all the selected studies and studies with unclear abstracts. In case the full text could not be accessed online, the corresponding authors were contacted. The collection of data and reviewing of the collected studies were performed by the first author (A.L). Additional reviewing and cross checking of findings were performed by the second author (M.C.) independently. Disagreements were resolved by discussion and consensus.

\section{Data extraction}

After the final study sample was determined, data from all studies were extracted into an Excel data sheet. This included: first author, year of publication, study design, number of subjects in both study and/or control group, age range and mean age of subjects and/or controls, used 3D imaging method, main subject and assessed syndrome(s). These data are structured in Table 2.

\section{Quality assessment}

A quality assessment of the included articles was conducted to evaluate their methodological quality. Therefore, the validated Methodological Index for Non-Randomized Studies (MINORS) was used (detailed in Table 3) ${ }^{65}$ This instrument was originally developed to review surgical research, where randomization is not always feasible. Studies on craniofacial syndromes are seldom randomized controlled trials (RCTs) due to ethical reasons and often have small study groups because of the low incidence of these syndromes. However, it was still useful to systematically review the existing literature and answer questions in that particular field. Taking into account all the above, 
Table 1 Search strategy

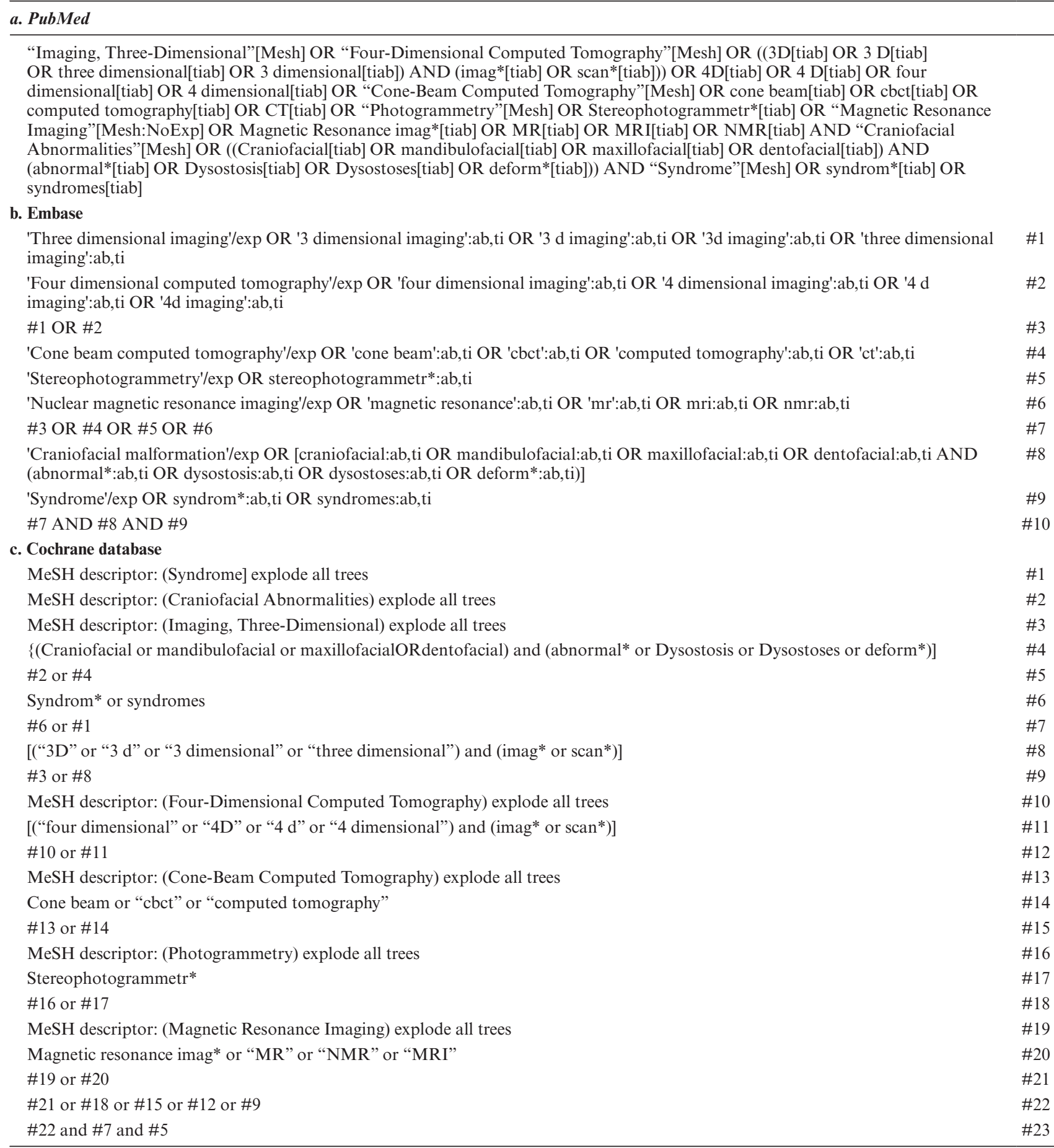

we considered the MINORS index as the most appropriate quality assessment index to evaluate the articles of this systematic review. According to this scale, the articles were divided into comparative and non-comparative studies with different scorings for both groups. Each item of the scale was given a score of 0 (not reported), 1 (reported but inadequate) or 2 (reported and adequate). For non-comparative studies, 8 items have to be scored, so the global ideal score is 16 , while for comparative studies, there are 4 additional items so, the global ideal score for comparative studies is 24 . The first author (A.L.) scored all the included articles and the second author (M.C.) was consulted in case of doubt. Judging of the used statistical analysis of the 


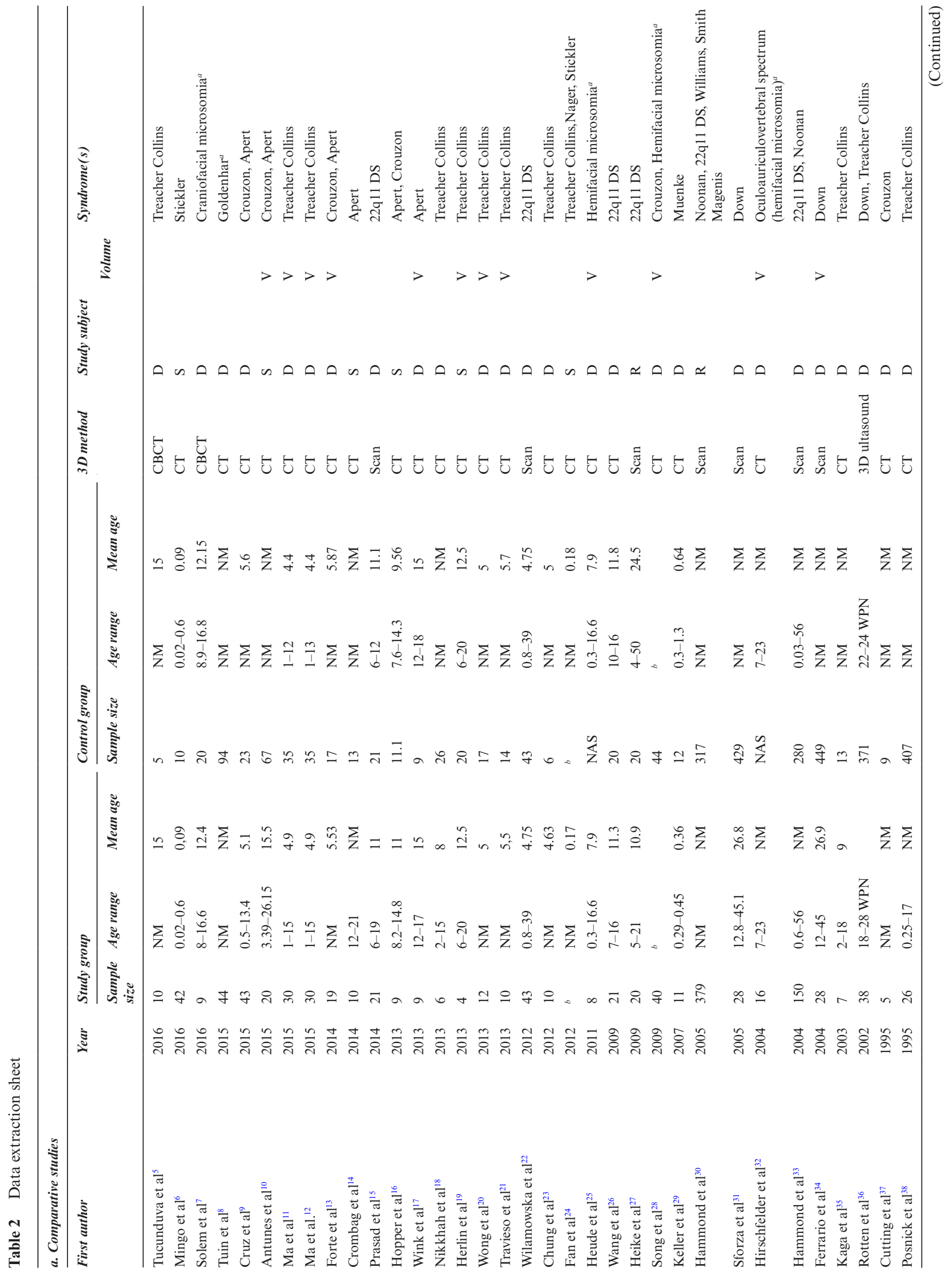




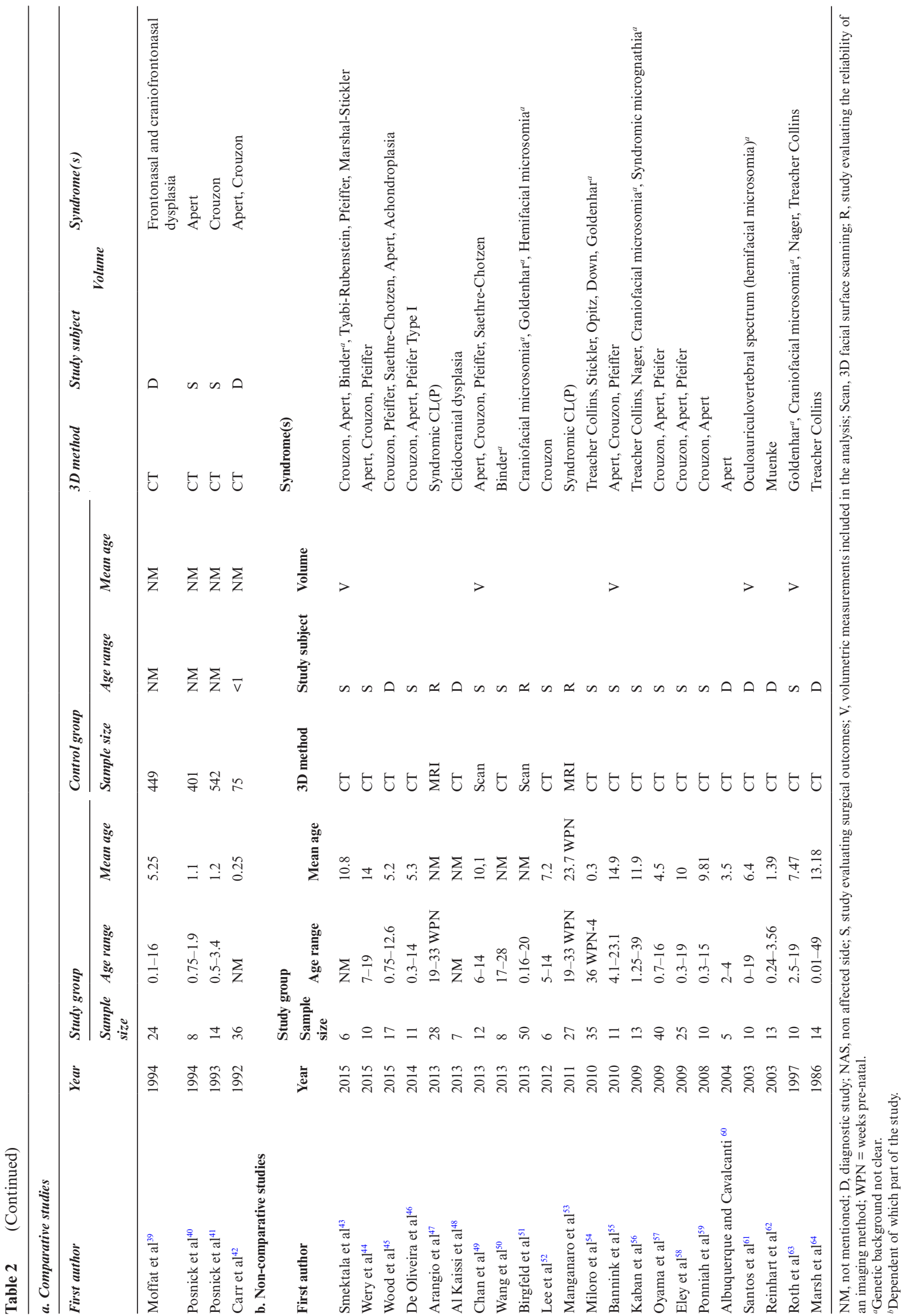


Table 3 MINORS

a. Scoring for comparative studies

\begin{tabular}{|c|c|c|c|c|c|c|c|c|c|c|c|c|c|c|}
\hline First author & Year & 1 & 2 & 3 & 4 & 5 & 6 & 7 & 8 & 9 & 10 & 11 & 12 & Score ${ }^{a}$ \\
\hline Tucunduva et $\mathrm{al}^{5}$ & 2016 & 2 & 1 & 1 & 2 & 1 & 2 & 0 & 0 & 1 & 1 & 0 & 2 & 13 \\
\hline Mingo et $\mathrm{a}^{6}$ & 2016 & 2 & 0 & 1 & 2 & 0 & 2 & 0 & 0 & 1 & 2 & 0 & 2 & 12 \\
\hline Solem etal ${ }^{7}$ & 2016 & 2 & 0 & 1 & 2 & 0 & 2 & 2 & 1 & 1 & 1 & 2 & 2 & 16 \\
\hline Tuin et $\mathrm{al}^{8}$ & 2015 & 2 & 2 & 1 & 2 & 0 & 2 & 0 & 0 & 1 & 2 & 1 & 2 & 15 \\
\hline Cruz et $\mathrm{al}^{9}$ & 2015 & 2 & 0 & 0 & 2 & 0 & 2 & 0 & 0 & 2 & 0 & 2 & 2 & 12 \\
\hline Antunes et $\mathrm{al}^{10}$ & 2015 & 2 & 1 & 1 & 2 & 0 & 2 & 0 & 0 & 2 & 0 & 0 & 2 & 12 \\
\hline Ma et $\mathrm{al}^{11}$ & 2015 & 2 & 0 & 1 & 2 & 2 & 2 & 0 & 0 & 2 & 0 & 2 & 2 & 15 \\
\hline Ma et $\mathrm{al}^{12}$ & 2015 & 2 & 1 & 1 & 2 & 2 & 2 & 0 & 0 & 2 & 0 & 2 & 2 & 16 \\
\hline Forte et $\mathrm{al}^{13}$ & 2014 & 2 & 0 & 1 & 2 & 1 & 2 & 0 & 1 & 2 & 0 & 2 & 2 & 15 \\
\hline Crombag et al ${ }^{14}$ & 2014 & 2 & 1 & 0 & 2 & 0 & 2 & 0 & 0 & 2 & 1 & 1 & 2 & 13 \\
\hline Prasad et al ${ }^{15}$ & 2014 & 1 & 1 & 2 & 2 & 1 & 2 & 0 & 0 & 2 & 2 & 1 & 2 & 16 \\
\hline Hopper et $\mathrm{al}^{16}$ & 2013 & 2 & 0 & 1 & 2 & 0 & 2 & 2 & 0 & 1 & 0 & 1 & 2 & 13 \\
\hline Wink et $\mathrm{al}^{17}$ & 2013 & 2 & 1 & 1 & 2 & 0 & 0 & 0 & 0 & 2 & 0 & 2 & 2 & 12 \\
\hline Nikkhah et al ${ }^{18}$ & 2013 & 2 & 1 & 0 & 2 & 0 & 2 & 1 & 0 & 2 & 1 & 2 & 2 & 15 \\
\hline Herlin et $\mathrm{al}^{19}$ & 2013 & 2 & 1 & 0 & 2 & 0 & 2 & 0 & 0 & 2 & 0 & 0 & 1 & 10 \\
\hline Wong et $\mathrm{al}^{20}$ & 2013 & 2 & 0 & 1 & 2 & 0 & 2 & 0 & 0 & 2 & 0 & 1 & 2 & 12 \\
\hline Travieso et $\mathrm{al}^{21}$ & 2013 & 2 & 2 & 1 & 2 & 2 & 2 & 0 & 0 & 2 & 0 & 2 & 2 & 17 \\
\hline Wilamowska et al ${ }^{22}$ & 2012 & 2 & 1 & 0 & 2 & 1 & 2 & 0 & 0 & 2 & 0 & 2 & 2 & 14 \\
\hline Chung et $\mathrm{al}^{23}$ & 2012 & 2 & 0 & 1 & 2 & 0 & 2 & 0 & 0 & 2 & 0 & 2 & 2 & 13 \\
\hline Fan et $\mathrm{al}^{24}$ & 2012 & 2 & 2 & 0 & 2 & 0 & 2 & 0 & 0 & 2 & 0 & 1 & 2 & 13 \\
\hline Heude et al ${ }^{25}$ & 2011 & 2 & 0 & 0 & 2 & 0 & 2 & 0 & 0 & 1 & 2 & 2 & 2 & 13 \\
\hline Wang et $\mathrm{al}^{26}$ & 2009 & 2 & 0 & 0 & 2 & 1 & 0 & 0 & 0 & 1 & 0 & 2 & 2 & 10 \\
\hline Heike et $\mathrm{al}^{27}$ & 2009 & 2 & 0 & 0 & 2 & 1 & 2 & 0 & 0 & 2 & 2 & 1 & 2 & 14 \\
\hline Song et $\mathrm{al}^{28}$ & 2009 & 2 & 0 & 0 & 2 & 1 & 2 & 0 & 0 & 2 & 0 & 2 & 2 & 13 \\
\hline Keller et $\mathrm{al}^{29}$ & 2007 & 2 & 0 & 0 & 2 & 1 & 2 & 0 & 0 & 1 & 0 & 1 & 2 & 11 \\
\hline Hammond et $\mathrm{al}^{30}$ & 2005 & 2 & 0 & 2 & 2 & 0 & 2 & 0 & 0 & 2 & 0 & 0 & 2 & 12 \\
\hline Sforza et $\mathrm{al}^{31}$ & 2005 & 2 & 0 & 1 & 2 & 0 & 2 & 0 & 0 & 2 & 1 & 2 & 2 & 14 \\
\hline Hirschfelder et al ${ }^{32}$ & 2004 & 2 & 0 & 0 & 2 & 0 & 0 & 0 & 0 & 1 & 2 & 2 & 2 & 11 \\
\hline Hammond et $\mathrm{al}^{33}$ & 2004 & 2 & 1 & 0 & 2 & 0 & 2 & 0 & 1 & 2 & 2 & 1 & 2 & 15 \\
\hline Ferrario et $\mathrm{al}^{34}$ & 2004 & 2 & 0 & 1 & 2 & 1 & 2 & 0 & 0 & 2 & 1 & 2 & 2 & 15 \\
\hline Kaga et al ${ }^{35}$ & 2003 & 2 & 0 & 0 & 2 & 0 & 2 & 0 & 0 & 2 & 1 & 1 & 0 & 10 \\
\hline Rotten et $\mathrm{al}^{36}$ & 2002 & 2 & 2 & 0 & 2 & 1 & 2 & 0 & 0 & 2 & 2 & 2 & 2 & 15 \\
\hline Cutting et $\mathrm{al}^{37}$ & 1995 & 2 & 0 & 0 & 2 & 0 & 2 & 0 & 0 & 2 & 1 & 2 & 2 & 13 \\
\hline Posnick et al ${ }^{38}$ & 1995 & 2 & 0 & 0 & 2 & 1 & 2 & 0 & 0 & 2 & 1 & 2 & 2 & 14 \\
\hline Moffat et $\mathrm{al}^{39}$ & 1994 & 2 & 0 & 0 & 2 & 1 & 2 & 0 & 0 & 2 & 1 & 2 & 1 & 13 \\
\hline Posnick et $\mathrm{al}^{40}$ & 1994 & 2 & 2 & 0 & 2 & 0 & 2 & 0 & 0 & 2 & 1 & 2 & 1 & 14 \\
\hline Posnick et $\mathrm{al}^{41}$ & 1993 & 2 & 2 & 0 & 2 & 0 & 2 & 0 & 0 & 2 & 0 & 1 & 2 & 13 \\
\hline Carr et $\mathrm{al}^{142}$ & 1992 & 2 & 1 & 1 & 2 & 1 & 2 & 1 & 0 & 2 & 2 & 2 & 2 & 18 \\
\hline \multicolumn{15}{|c|}{ b. Scoring for non-comparative studies } \\
\hline Smektala et $\mathrm{al}^{43}$ & 2015 & 2 & 0 & 1 & 2 & 2 & 2 & 0 & 0 & & & & & 9 \\
\hline Wery et $\mathrm{al}^{44}$ & 2015 & 2 & 1 & 1 & 2 & 1 & 2 & 0 & 0 & & & & & 9 \\
\hline Wood et al ${ }^{45}$ & 2015 & 1 & 1 & 1 & 2 & 0 & 2 & 0 & 0 & & & & & 7 \\
\hline De Oliveira et al $^{46}$ & 2014 & 1 & 2 & 1 & 2 & 0 & 2 & 2 & 0 & & & & & 10 \\
\hline Arangio et $\mathrm{al}^{47}$ & 2013 & 1 & 0 & 1 & 2 & 0 & 2 & 0 & 0 & & & & & 6 \\
\hline Al Kaissi et al ${ }^{48}$ & 2013 & 1 & 0 & 0 & 2 & 0 & 2 & 0 & 0 & & & & & 5 \\
\hline Chan et $\mathrm{al}^{49}$ & 2013 & 2 & 0 & 0 & 2 & 0 & 2 & 2 & 0 & & & & & 8 \\
\hline Wang et $\mathrm{al}^{50}$ & 2013 & 1 & 0 & 0 & 2 & 0 & 0 & 0 & 0 & & & & & 3 \\
\hline Birgfeld et $a^{51}$ & 2013 & 2 & 2 & 0 & 2 & 2 & 2 & 0 & 0 & & & & & 10 \\
\hline
\end{tabular}


Table 3 (Continued)

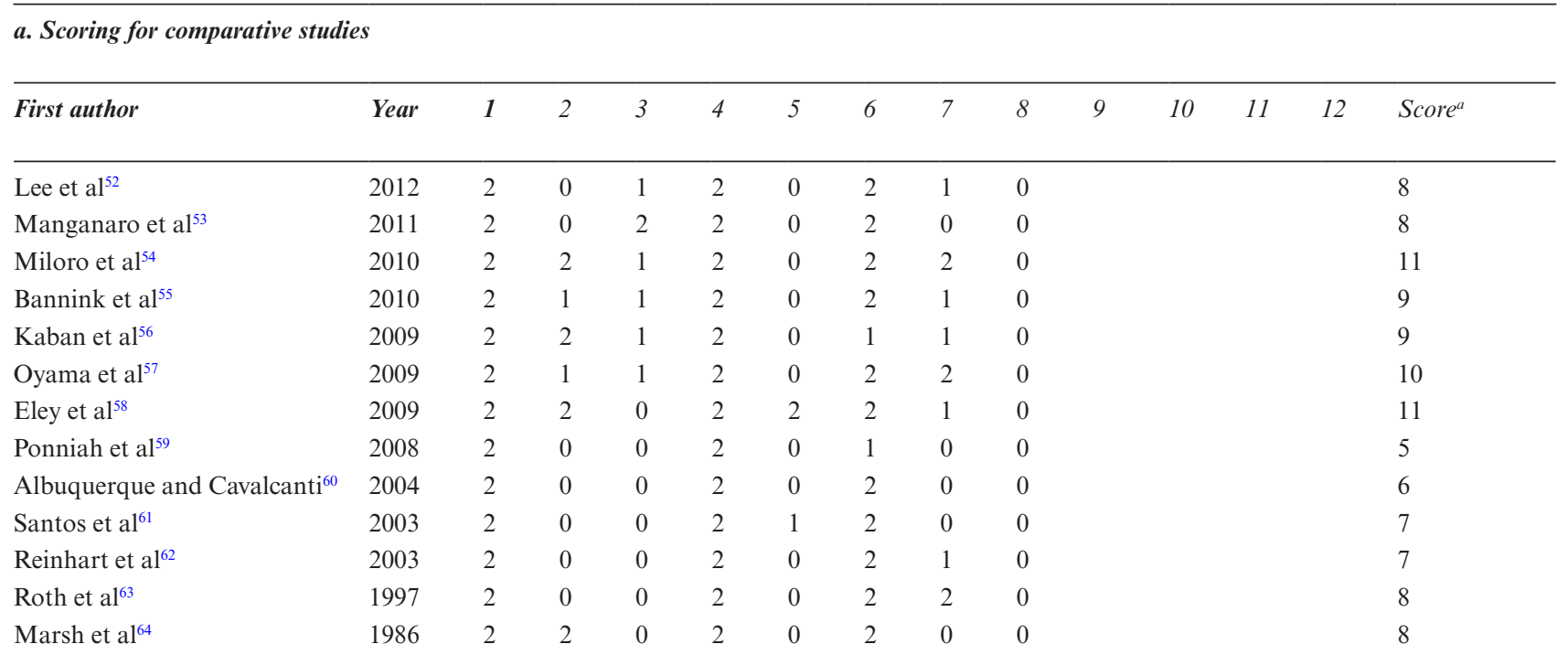

Correspondence of the numbers (taken from Slim et al): ${ }^{3}$, a clearly stated aim; 2, inclusion of consecutive patients; 3 , prospective collection of data; 4, endpoints appropriate to the aim of the study; 5 , unbiased assessment of the study endpoint; 6, follow-up period appropriate to the aim of the study; 7 , loss to follow up less than $5 \% ; 8$, prospective calculation of the study size. Additional criteria in the case of comparative studies: 9 , an adequate control group; 10, contemporary groups; 11, baseline equivalence of groups; 12, adequate statistical analyses.

${ }^{a}$ The items are scored 0 (not reported), 1 (reported but inadequate) or 2 (reported and adequate). The global ideal score being 16 for noncomparative studies and 24 for comparative studies.

comparative studies was performed by the two main reviewers (A.L and M.C), consulting a professional statistician in case of doubt.

\section{Results}

\section{Study selection}

The electronic search of PubMed, EMBASE and Cochrane Library yielded 2226 citations. Two additional records were found through other sources. After removing duplicates and selection on titles and abstracts, the full text of the 116 remaining articles was assessed. Reasons for excluding studies after full text reading were: facial structures not included in the analysis, only 2D imaging, articles were case reports, reviews or opinions. The final selection comprised 60 articles which were summarized in Table 2. The PRISMA flow diagram is shown in Figure 1.

\section{Study characteristics}

The assessment of the methodological quality revealed that there were 22 non-comparative and 38 comparative studies with a median MINORS score of 8 over 16 (7-9, 25th-75th percentile) and 13 over 24 (12-15, 25-75th percentile) for both groups respectively (Table 3 ). Topics that were consistently scored as "not reported" in the majority of the studies were Item 7 "loss to follow-up less than 5\%" and Item 8 "prospective calculation of the study size". However, when a follow-up period was not included in a study but was also not required to meet the aim of that study, Item 6 of the scale was scored "reported and adequate" and Item 7 was scored as "not reported" which contributed to the low scores on Item 7. Of the comparative studies, 76\% (29/38) had an adequate control group. When the non-affected side or a historical control group was used for control, this was scored as inadequate.

The aim and outcome of the articles were heterogeneous, but three general subject matters could be distinguished: controlled and non-controlled diagnostic studies, surgical outcomes and evaluation of imaging techniques. More than half of the publications $(34 / 60,57 \%)$ could be considered as diagnostic studies describing phenotypes and craniofacial features in patients with craniofacial syndromes, often in comparison with healthy controls. About one third of the articles $(21 / 60,35 \%)$ was related to surgical outcomes or the description of surgical techniques. Distraction osteogenesis was the most evaluated surgical procedure in this group $(17 / 21,81 \%)$.

The last and smallest group (5/60,8\%) contained articles relating to the reliability or usefulness of specific imaging methods such as MRI and facial surface scanning. In two publications of the same study group, the ability of MRI to detect craniofacial anomalies in foetuses was pointed out and compared with standard 2D ultrasound examination. ${ }^{47,53}$ In three other publications, facial surface scanning was the used imaging method. The reliability of the $3 \mathrm{dMD}$ Mace system (3dMD, Atlanta, GA) in patients with 22q11.2 deletion syndrome in comparison with direct calipre measurements was 

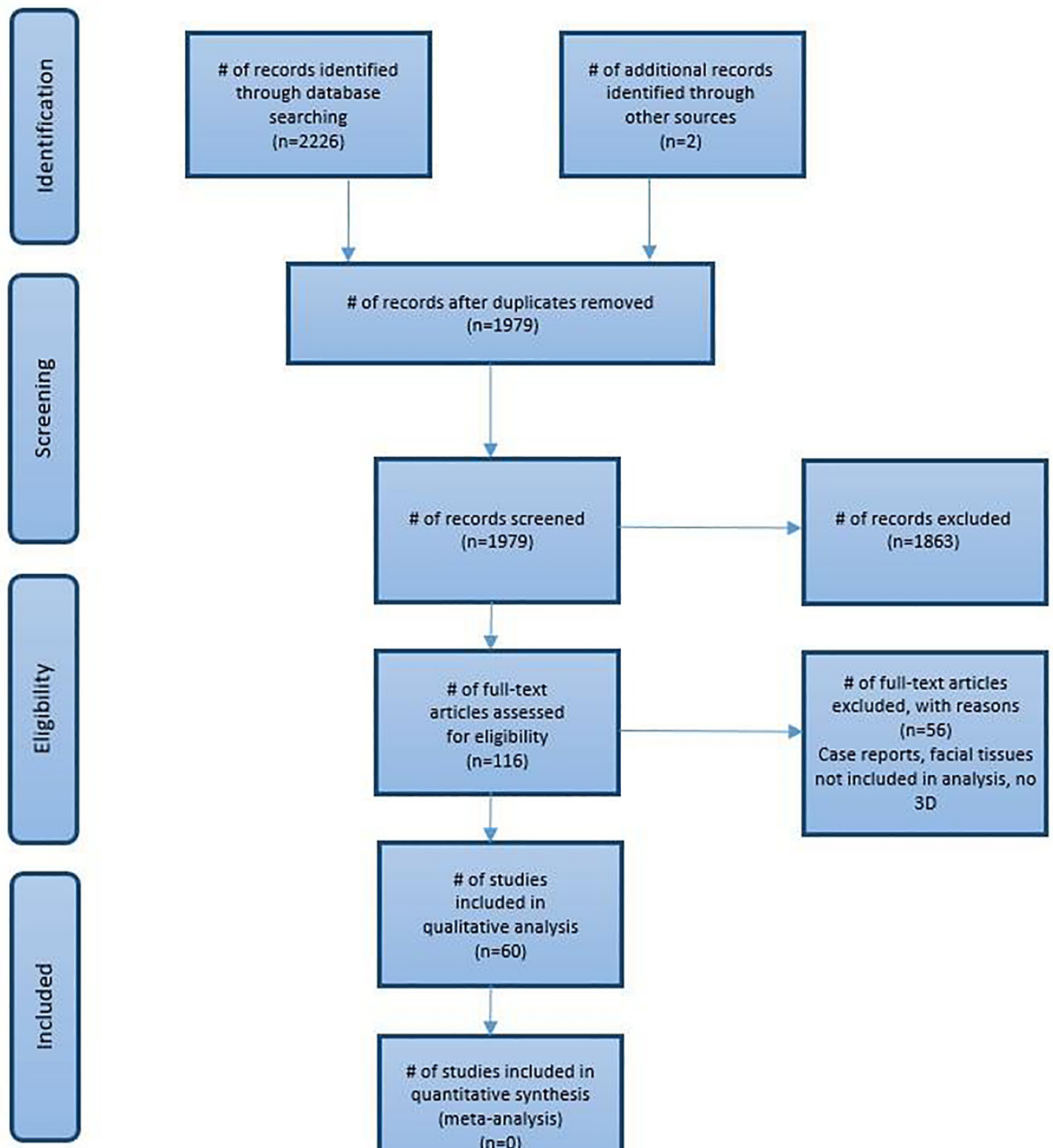

\# of studies

included in

qualitative analysis $(n=60)$

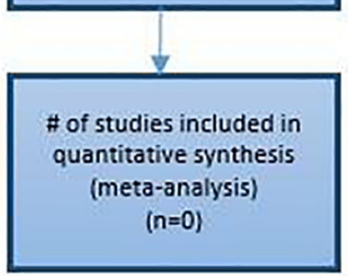

\# of additional records identified through other sources $(n=2)$

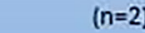


full face for control-syndrome and syndrome-syndrome comparisons for children ranged from 87 to $-97 \%$. It was suggested that visualizations of $3 \mathrm{D}$ face-shape variations could be used to train physicians to recognize the key components of particular syndromes.

In the majority of the included studies $(46 / 60,77 \%)$, $\mathrm{CT}$ was used as the imaging modality. Other methods used were: facial surface scanning $(9 / 60,15 \%)$, MRI $(2 / 60,3 \%)$, CBCT $(2 / 60,3 \%)$ and ultrasonography $(1 / 60$, $2 \%)$.

In 17 out of 60 studies $(28 \%)$, volumes were calculated as part of the analysis. 11 of these studies were diagnostic studies in which the volume of a certain anatomical structure like the condylar head, maxillary sinus, muscles or even the entire maxillary or mandibular body was calculated and compared with a control group. In the other six studies, volumes were calculated before and after maxillofacial surgery. The studies are indicated with a "V" in the column "Volume" of Table 2.

Three craniofacial syndromes were by far more studied than others, namely: Treacher Collins, Crouzon and Apert syndrome in 16, 18 and 18 studies, respectively. In 19 out of the 21 surgical outcome studies $(90 \%)$, patients with one of these syndromes were included. In the other studies, conditions were sometimes included which have not yet been clearly described in literature as genetically based syndromes but they were, however, treated as such and were often studied together with other known syndromes. This was the case in 12 of the 60 studies $(20 \%)$ and the conditions included were: hemifacial microsomia and Goldenhar syndrome, craniofacial microsomia, Binder syndrome and syndromic micrognatia. These studies are identified in Table 2 with the letter (a).

The median of the reported mean ages of patients was 7.3 years $(4.8-11.2,25-75$ th percentile). However, the age range of patients both within and between studies was often wide, ranging from only a few weeks to adulthood. Three studies focussed on prenatal diagnosis of craniofacial anomalies and, therefore, foetuses in the third trimester where included in these studies. Also for sample size, a large variability between the studies existed, ranging from 4 to 375 subjects in the study group.

The included studies were published between 1986 and 2016. With regard to evolutions of the included studies over time, the following trends were noticed (reflected in Figures 2 and 3 and Table 2):

- There was an increase in the amount of studies with time (Figure 2).

- CT was the imaging method most often used, although from 2002 onwards, other modalities were involved, of which non-invasive facial surface scanning was described most frequently (Figure 3 ).

As a result of the information gathered from the included studies, a number of considerations to improve

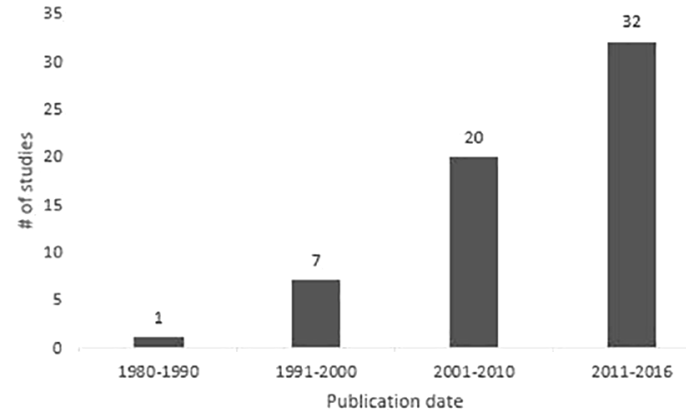

Figure 2 Number of studies per publication period.

the methodological quality and reporting of future research projects have been summarized in Table 4 and are discussed with more detail in the "Discussion" section.

\section{Discussion}

The result of this systematic review was a heterogeneous collection of articles about 3D imaging in patients with craniofacial syndromes of moderate methodological quality, as the median MINORS scores were only 50 and $54 \%$ of the maximum scores. The low incidence of craniofacial syndromes makes it difficult to build up large study groups and randomized controlled trials are often not possible owing to ethical reasons. Also, the absence of blinding and a lack of information on dropouts contributed to the moderate scores on the MINORS scale. For comparative studies, control groups of healthy subjects, matched for age, gender and ethnicity were considered as the reference standard for comparison (score 2 on Item 9 of the MINORS scale). This was the case in 29 studies $(29 / 38,76 \%)$. In two studies on hemifacial microsomia, the non-affected side was used for comparison. ${ }^{25,32}$ This provided control for confounding environmental factors; however, there may have been unknown influences of the genetic condition on the non-affected side, resulting in a score 1 ("reported but inadequate") on Item 9 ("adequate control group") of the MINORS scale. Some studies made use of a historical control group, previously described in

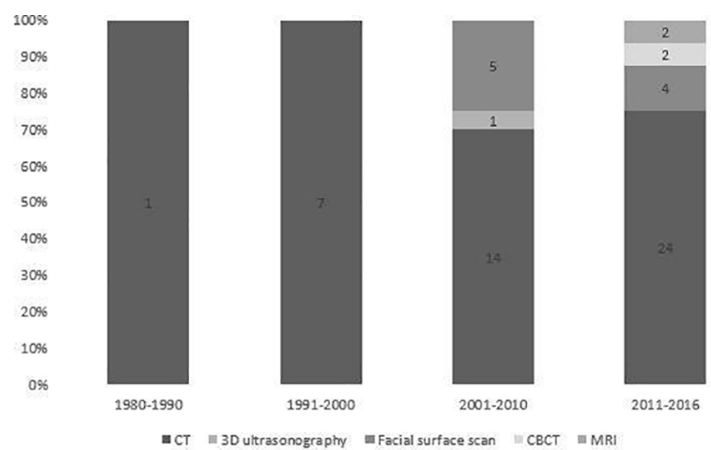

Figure 3 Imaging method per publication period. 
Table 4 Recommendations for future research

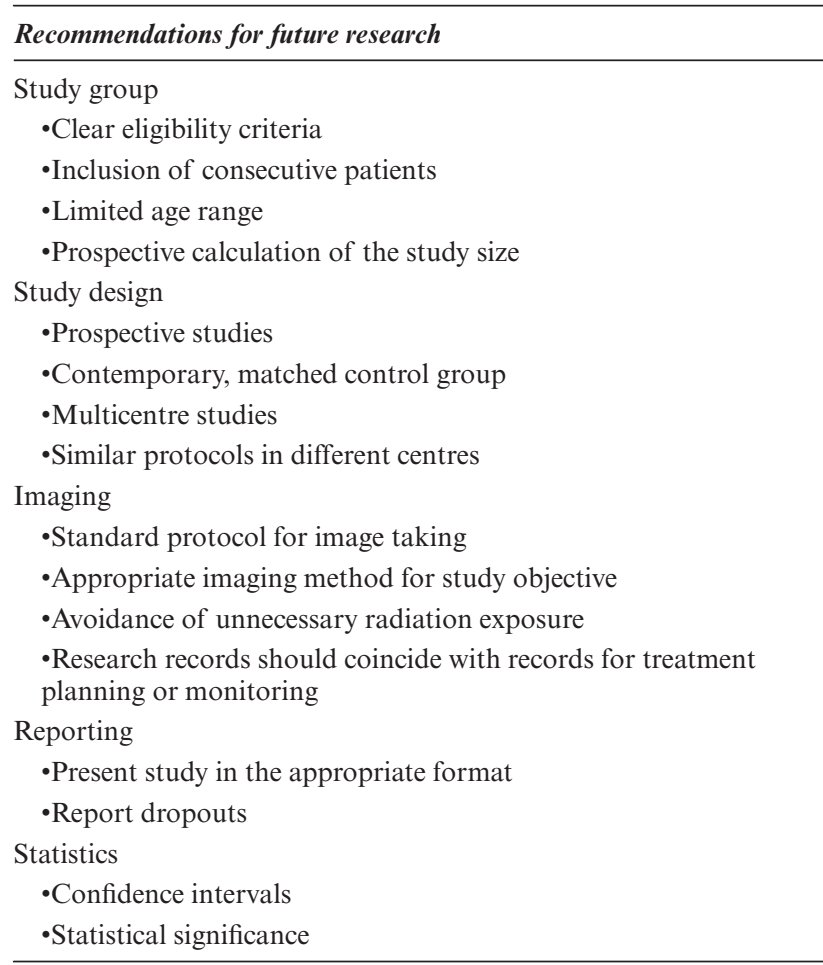

literature. This also was considered as inadequate on the MINORS scale because there is no control over the different variables that could be affecting its comparability and, therefore, there is no way of controlling the possible bias.

CT was the imaging method most often used in the past as well as in recent studies. Of all methods available to produce 3D images of the craniofacial area, CT has the highest radiation exposure. Young individuals are especially vulnerable to the harmful effects of X-rays, so adaptation of the projection and radiation exposure according to the area of interest and age of the patient is mandatory. Depending on what information is required, less-invasive $\mathrm{CBCT}$ allows for a significant reduction in radiation exposure compared with conventional $\mathrm{CT}^{66}$ CBCT renders 3D images of the craniofacial area and is mainly used in the fields of dentistry, maxillofacial surgery and otorhinolaryngology. In this review, only one article was included in which CBCT was used (to investigate the area of the mandible between the mental foramina). ${ }^{5}$

Non-radiation methods are undoubtedly preferable over invasive methods but they have their specific indications. 3D facial surface scanning was the second most used imaging method in the studies of this review. It provides highly accurate images of the external facial morphology so this could be an appropriate method for diagnostic purposes as well as to evaluate facial soft tissue changes after surgery. ${ }^{27,67}$ The fact that it is more and more used by researchers and clinicians shows a positive trend towards a reduction in the use of radiation methods.

Images can be evaluated both qualitatively and quantitatively by performing measurements on them. Besides, 3D images have the unique advantage of allowing for volumetric measurements. In total, there were 17 studies with volumetric measurements $(17 / 60$, $28 \%$ ). In the group of diagnostic studies, volumes were calculated in 11 studies $(11 / 34,32 \%)$. In the remaining 23 studies, images were used to perform 2D linear or angular measurements or to assess asymmetries, atrophy or aplasia of certain elements. Depending on what information is needed, the least invasive imaging method should be selected. For instance, if only skeletal measurements need to be made in the sagittal plane, it would be more appropriate to take a conventional lateral cephalogram than a CT, as one could wonder what is the real advantage in using only $2 \mathrm{D}$ measurements in a $3 \mathrm{D}$ projection. ${ }^{23,26}$

The subject matters of the included articles could be divided in three major groups: controlled and non-controlled diagnostic studies, evaluation of surgical outcomes and evaluation of 3D imaging methods.

The largest group contains diagnostic studies, where aims were highly variable and go from a general description of the facial area in a certain craniofacial syndrome to a detailed measuring of a specific anatomical structure, such as the masseteric muscle or the condylar head. Sixty-five percent $(22 / 34,65 \%)$ of studies in this group were comparative, quantitative studies. Diagnostic studies without a control group $(6 / 34,18 \%)$ can only attempt to describe the craniofacial phenotype in the studied population. Especially when sample sizes are small, care should be taken in extrapolating the results to a larger group.

A second group existed of articles relating to surgical interventions. The deformity of the craniofacial skeleton can be very complex and severe in patients with craniofacial syndromes and correction is endeavoured using advancing procedures. Distraction osteogenesis in early childhood is often performed for this purpose. In 17 out of 21 articles in this group (81\%), distraction osteogenesis was evaluated with $3 \mathrm{D}$ imaging. The imaging method was CT in all studies except one. Images were taken prior to surgery to prepare the surgical procedure and to measure pre-operative values. After surgery, a follow-up image was often taken as well to perform post-operative measurements and evaluate the resulting anatomy in an objective way. By postponing the second image to a moment later in time when the results of the surgery are thought to be stable rather than taking an image immediately after the distraction has ended, a third image for follow up could be avoided and radiation exposure would, therefore, be further reduced. Again, 
when the mean age of subjects is only a few weeks or months old, this certainly needs to be taken into account. One surgical evaluation study did successfully assess soft and hard tissue gain after distraction osteogenesis using facial surface scanning. ${ }^{49}$

The third group contained articles in which the reliability of an imaging method was tested. Such articles can be of great value to provide scientific evidence about new imaging and measuring techniques.

Treacher Collins, Apert and Crouzon were the syndromes most studied. Interestingly, nearly all studies concerning surgical interventions $(19 / 21,90 \%)$ were performed in patients with one of these syndromes. This could be owing to the extensively aberrant morphology associated with these syndromes demanding one or more surgical interventions.

The genetic background of these three syndromes is well known. Owing to the rapidly progressing developments in the field of human genetics, even more information becomes available. However, this is not the case for other less frequently studied conditions, such as hemifacial microsomia or Goldenhar syndrome, where the underlying genetic origin is far less clear. In our study, we included genetically based, congenital syndromes with oral and craniofacial manifestations and we chose to exclude conditions such as non-syndromic oral clefts or OSAS because of them being genetically based but non-syndromic or non-syndromic but genetically based. Owing to the lack of knowledge and need for further investigation of these " unlabelled" conditions, it remains to be discussible which of them can be regarded as craniofacial syndromes and which not, a fact that could be considered as a limitation of this systematic review.

Further genetic research may help to clarify this. The aim of this review was to collect evidence about the use and developments of $3 \mathrm{D}$ imaging methods in patients with congenital anomalies. By including a wide range of diseases, our intent was not to miss valuable information.

Owing to the limited amount and large heterogeneity of the available literature, we were not able to perform statistical tests on the gathered data. Therefore, the focus lied on the methodological quality of the articles and on describing and summarizing the information of the different studies.

\section{Remarks for future research}

To allow for a more detailed and meta-analytic evaluation of the scientific literature about this topic, a significant improvement in study quality is necessary and could be achieved if some aspects of research would become more standard. We attempted to formulate some recommendations with the aim of improving future research and care of patients with craniofacial syndromes (summarized in Table 4). These recommendations were based on the MINORS scale and on the Eurocleft consensus guidelines ${ }^{68}$ about imaging. The
Eurocleft project was held between 1996 and 2000 and its major aim was to improve the effectiveness and efficiency of care for European children with cleft lip/ palate. To do so, information about treatment protocols of different European cleft teams was gathered and subsequently transformed into guidelines describing minimum standards for care of patients with clefts. A specific topic about record taking was also part of the Eurocleft report and this we now applied to patients with craniofacial syndromes.

Regarding the study group, clear eligibility criteria should be formulated. All patients potentially fitting for inclusion should be included, so selection bias could be minimized. On the other hand, age range should be limited because of its influence on study results, especially when sample sizes are small. For example, when a study group consists of children as well as adults, the influence of growth is difficult to incorporate when outcomes are generalized. In that perspective, the study aim should be adapted to the age range of patients. To empower the significance of study results, a prospective calculation of study size is mandatory. To reach a substantial group of patients within a limited age range and taking into account the low incidences of craniofacial syndromes it will be necessary to set up multicentre studies. In this way, data obtained following similar protocols can be gathered. This is why prospective trials are much preferred over retrospective studies, since standard protocols can be set in advance and followed within one and between different centres to augment the comparability of outcomes. Additionally, results of the study group should be compared with a contemporary, matched control group ideally composed of non-affected individuals.

Also, for the image taking itself, it is important that a protocol exists and is followed by every person who takes an image. In this way, the comparability and quality of the obtained images will be higher, retakes will be much less needed and there will be less missing data because of bad image quality. Before taking an image, it should be clear what it will be used for so the appropriate imaging method can be selected. In case of radiological images, the field of view and exposure time should also be adjusted to avoid excessive radiation exposure.

Children with craniofacial syndromes are often treated by practitioners of several disciplines and go through many interventions and surgical procedures from an early age. Without a doubt, images can thereby be very helpful. However, the need for these images should match as much as possible between different professionals, which should be a priority of the multidisciplinary team. For the patient, in particular, this would be beneficial because radiation exposure, amount of visits and additional burden would be reduced. Subsequently, records for research goals should coincide with records for treatment planning or monitoring as well.

Concerning the reporting of a study, dropouts and subjects that are lost during follow up should be 
mentioned. Furthermore, the study should be presented in the appropriate format. Mainly, the distinction between a case series and a clinical trial should be clear. Last, the statistical analysis of results should not be forgotten, and confidence intervals and statistical significance should be provided when possible.

\section{Conclusion}

In this systematic review about 3D imaging of facial soft and hard tissues in children with craniofacial syndromes 60 articles were included. The overall methodological quality of the articles was moderate, indicated by median MINORS scores around 50\% of the maximum score. Main subjects of the included articles were diagnostic studies, evaluation of surgical outcomes and evaluation of 3D imaging methods. Studies showed a large heterogeneity in sample size, age range and study design, making statistical analysis not possible. Even though randomization and blinding are often difficult in this domain, other measures could be taken to improve the quality of future research as proposed in the present article. Collaboration between different disciplines and even different centres would be the key component to accomplish this together with standard protocols for image taking and study set up.

\section{References}

1. Smeets D, Claes P, Vandermeulen D, Clement JG. Objective 3D face recognition: evolution, approaches and challenges. Forensic Sci Int 2010; 201: 125-32. doi: https://doi.org/10.1016/j.forsciint. 2010.03.023

2. Sforza C, de Menezes M, Ferrario V. Soft- and hard-tissue facial anthropometry in three dimensions: what's new. J Anthropol Sci 2013; 91: 159-84. doi: https://doi.org/10.4436/jass.91007

3. Moher D, Liberati A, Tetzlaff J, Altman DG, PRISMA Group. Preferred reporting items for systematic reviews and meta-analyses: the PRISMA statement. Int J Surg 2010; 8: 336-41. doi: https://doi.org/10.1016/j.ijsu.2010.02.007

4. Guyatt G, Drummond R, Meade M, Cook D. The evidence based-medicine working group users' guides to the medical literature. 2nd ed. Chicago: McGraw Hill; 2008.

5. Mingo KM, Sidman JD, Sampson DE, Lander TA, Tibesar RJ, Scott AR. Use of external distractors and the role of imaging prior to mandibular distraction in infants with isolated pierre robin sequence and stickler syndrome. JAMA Facial Plast Surg 2016; 18: 95-100. doi: https://doi.org/10.1001/jamafacial.2015. 1658

6. Solem RC, Ruellas A, Miller A, Kelly K, Ricks-Oddie JL, Cevidanes L. Congenital and acquired mandibular asymmetry: mapping growth and remodeling in 3 dimensions. Am J Orthod Dentofacial Orthop 2016; 150: 238-51. doi: https://doi.org/10. 1016/j.ajodo.2016.02.015

7. Tuin J, Tahiri Y, Paliga JT, Taylor JA, Bartlett SP. Distinguishing goldenhar syndrome from craniofacial microsomia. J Craniofac Surg 2015; 26: 1887-92. doi: https://doi.org/10.1097/SCS. 0000000000002017

8. Cruz AA, Garcia DM, Akaishi PM, Arnaud E, Milbratz GH, Bertrand AL. Globe protrusion and interorbital divergence in syndromic faciocraniosynostoses. J Craniofac Surg 2015; 26: 1163-4. doi: https://doi.org/10.1097/SCS.0000000000001553

11. Ma X, Forte AJ, Berlin NL, Alonso N, Persing JA, Steinbacher DM. Reduced three-dimensional nasal airway volume in treacher collins syndrome and its association with craniofacial morphology. Plast Reconstr Surg 2015; 135: 885e-94. doi: https:// doi.org/10.1097/PRS.0000000000001160

12. Ma X, Forte AJ, Persing JA, Alonso N, Berlin NL, Steinbacher DM. Reduced three-dimensional airway volume is a function of skeletal dysmorphology in Treacher Collins syndrome. Plast Reconstr Surg 2015; 135: 382e-92. doi: https://doi.org/10. 1097/PRS.0000000000000993

13. Forte AJ, Alonso N, Persing JA, Pfaff MJ, Brooks ED, Steinbacher DM. Analysis of midface retrusion in crouzon and apert syndromes. Plast Reconstr Surg 2014; 134: 285-93. doi: https://doi.org/10.1097/PRS.0000000000000360

14. Crombag GA, Verdoorn MH, Nikkhah D, Ponniah AJ, Ruff C, Dunaway D. Assessing the corrective effects of facial bipartition

distraction in Apert syndrome using geometric morphometrics. $J$ Plast Reconstr Aesthet Surg 2014; 67: e151-e161. doi: https://doi. org/10.1016/j.bjps.2014.02.019

15. Prasad S, Katina S, Hennessy RJ, Murphy KC, Bowman AW, Waddington JL. Craniofacial dysmorphology in 22q11.2 deletion syndrome by $3 \mathrm{D}$ laser surface imaging and geometric morphometrics: Illuminating the developmental relationship to risk for psychosis. Am J Med Genet A 2015; 167: 529-36. doi: https://doi. org/10.1002/ajmg.a.36893

16. Hopper RA, Kapadia H, Morton T. Normalizing facial ratios in apert syndrome patients with Le Fort II midface distraction and simultaneous zygomatic repositioning. Plast Reconstr Surg 2013; 132: 129-40. doi: https://doi.org/10.1097/PRS. 0b013e318290fa $8 \mathrm{a}$

17. Wink JD, Bastidas N, Bartlett SP. Analysis of the longterm growth of the mandible in apert syndrome. J Craniofac Surg 2013; 24: 1408-10. doi: https://doi.org/10.1097/SCS. 0b013e31828dcf09

18. Nikkhah D, Ponniah A, Ruff C, Dunaway D. Planning surgical reconstruction in treacher-collins syndrome using virtual simulation. Plast Reconstr Surg 2013; 132: 790e-805. doi: https://doi.org/ 10.1097/PRS.0b013e3182a48d33

19. Herlin C, Doucet JC, Bigorre M, Captier G. Computerassisted midface reconstruction in Treacher Collins syndrome part 2: soft tissue reconstruction. J Craniomaxillofac Surg 2013; 41: 676-80. doi: https://doi.org/10.1016/j.jcms.2013.01. 008

20. Wong KR, Pfaff MJ, Chang CC, Travieso R, Steinbacher DM. A range of malar and masseteric hypoplasia exists in treacher collins syndrome. J Plast Reconstr Aesthet Surg 2013; 66: 43-6. doi: https://doi.org/10.1016/j.bjps.2012.07.028

21. Travieso R, Chang CC, Terner JS, Beckett J, Wong K, Teng E, et al. A range of condylar hypoplasia exists in treacher collins syndrome. J Oral Maxillofac Surg 2013; 71: 393-7. doi: https:// doi.org/10.1016/j.joms.2012.04.031

22. Wilamowska K, Wu J, Heike C, Shapiro L. Shape-based classification of 3D facial data to support 22q11.2DS craniofacial research. J Digit Imaging 2012; 25: 400-8. doi: https://doi.org/10. 1007/s10278-011-9430-x

23. Chung MT, Levi B, Hyun JS, Lo DD, Montoro DT, Lisiecki J, et al. Pierre robin sequence and treacher collins hypoplastic mandible comparison using three-dimensional morphometric analysis. $J$ Craniofac Surg 2012; 23(7 Suppl 1): S17-S21. doi: https://doi.org/ 10.1097/SCS.0b013e318258bcf1

24. Fan K, Andrews BT, Liao E, Allam K, Raposo Amaral CA, Bradley JP. Protection of the temporomandibular joint during syndromic neonatal mandibular distraction using condylar unloading. Plast Reconstr Surg 2012; 129: 1151-61. doi: https:// doi.org/10.1097/PRS.0b013e31824a2d00 
25. Heude E, Rivals I, Couly G, Levi G. Masticatory muscle defects in hemifacial microsomia: a new embryological concept. Am J Med Genet A 2011; 155A: 1991-5. doi: https://doi.org/10.1002/ajmg.a. 34095

26. Wang K, Yang Y, Shen F, Tao J, Xu H, Portnof JE, et al. Utilization of three-dimensional computed tomography for craniofacial phenotypic analysis in children with velocardiofacial syndrome. J Craniofac Surg 2009; 20: 2013-9. doi: https://doi.org/10.1097/ SCS.0b013e3181bd2e34

27. Heike CL, Cunningham ML, Hing AV, Stuhaug E, Starr JR. Picture perfect? Reliability of craniofacial anthropometry using three-dimensional digital stereophotogrammetry. Plast Reconstr Surg 2009; 124: 1261-72. doi: https://doi.org/10.1097/PRS. 0b013e3181b454bd

28. Song SY, Hong JW, Roh TS, Kim YO, Kim DW, Park BY. Volume and distances of the maxillary sinus in craniofacial deformities with midfacial hypoplasia. Otolaryngol Head Neck Surg 2009; 141: 614-20. doi: https://doi.org/10.1016/j.otohns.2009.08.018

29. Keller MK, Hermann NV, Darvann TA, Larsen P, Hove HD, Christensen L, et al. Craniofacial morphology in muenke syndrome. J Craniofac Surg 2007; 18: 374-86. doi: https://doi.org/10.1097/ scs.0b013e31803ffa63

30. Hammond P, Hutton TJ, Allanson JE, Buxton B, Campbell LE, Clayton-Smith J, et al. Discriminating power of localized three-dimensional facial morphology. Am J Hum Genet 2005; 77: 999 1010. doi: https://doi.org/10.1086/498396

31. Sforza C, Dellavia C, Dolci C, Donetti E, Ferrario VF. A quantitative three-dimensional assessment of abnormal variations in the facial soft tissues of individuals with Down syndrome. Cleft Palate Craniofac J 2005; 42: 410-6. doi: https://doi.org/10.1597/ 04-005.1

32. Hirschfelder U, Piechot E, Schulte M, Leher A. Abnormalities of the TMJ and the musculature in the oculo-auriculo-vertebral spectrum (OAV). A CT study. J Orofac Orthop 2004; 65: 204-16. doi: https://doi.org/10.1007/s00056-004-0331-1

33. Hammond P, Hutton TJ, Allanson JE, Campbell LE, Hennekam RC, Holden S, et al. 3D analysis of facial morphology. Am J Med Genet A 2004; 126A: 339-48. doi: https://doi.org/10. 1002/ajmg.a.20665

34. Ferrario VF, Dellavia C, Colombo A, Sforza C. Three-dimensional assessment of nose and lip morphology in subjects with down syndrome. Ann Plast Surg 2004; 53: 577-83. doi: https://doi. org/10.1097/01.sap.0000130702.51499.6b

35. Kaga K, Takegoshi H, Yamasoba T, Nakamura M, Kaneko M, Ino K. Aplasia of zygomatic arch and dislocation of temporomandibular joint capsule in treacher-collins syndrome: three-dimensional reconstruction of computed tomographic scans. Int $J$ Pediatr Otorhinolaryngol 2003; 67: 1189-94. doi: https://doi.org/ 10.1016/j.ijporl.2003.07.001

36. Rotten D, Levaillant JM, Martinez H, Ducou le Pointe H, Vicaut E. The fetal mandible: a 2D and 3D sonographic approach to the diagnosis of retrognathia and micrognathia. Ultrasound Obstet Gynecol 2002; 19: 122-30. doi: https://doi.org/10.1046/j. 0960-7692.2001.00622.x

37. Cutting C, Dean D, Bookstein FL, Haddad B, Khorramabadi D, Zonneveld FW, et al. A three-dimensional smooth surface analysis of untreated Crouzon's syndrome in the adult. J Craniofac Surg 1995; 6: 444-53. doi: https://doi.org/10.1097/00001665199511000-00004

38. Posnick JC, al-Qattan MM, Moffat SM, Armstrong D. Cranio-orbito-zygomatic measurements from standard CT scans in unoperated treacher collins syndrome patients: comparison with normal controls. Cleft Palate Craniofac J 1995; 32: 20-4. doi: https://doi.org/10.1597/1545-1569(1995)032<0020:CMFSC $\mathrm{S}>2.3 . \mathrm{CO} ; 2$

39. Moffat SM, Posnick JC, Pron GE, Armstrong DC. Frontonasal and craniofrontonasal dysplasia: preoperative quantitative description of the cranio-orbito-zygomatic region based on computed and conventional tomography. Cleft Palate Craniofac J 1994; 31: 97-105. doi: https://doi.org/10.1597/15451569(1994)031<0097:FACDPQ>2.3.CO;2
40. Posnick JC, Lin KY, Jhawar BJ, Armstrong D. Apert syndrome: quantitative assessment by $\mathrm{CT}$ scan of presenting deformity and surgical results after first-stage reconstruction. Plast Reconstr Surg 1994; 93: 489-97.

41. Posnick JC, Lin KY, Jhawar BJ, Armstrong D. Crouzon syndrome: quantitative assessment of presenting deformity and surgical results based on CT scans. Plast Reconstr Surg 1993; 92: 1027-37.

42. Carr M, Posnick JC, Pron G, Armstrong D. Cranio-orbito-zygomatic measurements from standard CT scans in unoperated crouzon and apert infants: comparison with normal controls. Cleft Palate Craniofac J 1992; 29: 129-36. doi: https://doi.org/10. 1597/1545-1569(1992)029<0129:COZMFS >2.3.CO;2

43. Smektala T, Nysjö J, Thor A, Homik A, Sporniak-Tutak K, Safranow K, et al. Three-dimensional eyeball and orbit volume modification after LeFort III midface distraction. J Craniofac Surg 2015; 26: 1652-5. doi: https://doi.org/10.1097/SCS. 0000000000001601

44. Wery MF, Nada RM, van der Meulen JJ, Wolvius EB, Ongkosuwito EM. Three-dimensional computed tomographic evaluation of Le Fort III distraction osteogenesis with an external device in syndromic craniosynostosis. Br J Oral Maxillofac Surg 2015; 53: 285-91. doi: https://doi.org/10.1016/j.bjoms.2014.12.016

45. Wood BC, Oh AK, Keating RF, Boyajian MJ, Myseros JS, Magge SN, et al. Progressive postnatal pansynostosis: an insidious and pernicious form of craniosynostosis. J Neurosurg Pediatr 2015; 16: 309-16. doi: https://doi.org/10.3171/2015.1.PEDS14464

46. De Oliveira RS, Ando A, Santos MV, Marques-Neto PB, Machado HR. Craniofacial distraction osteogenesis with rigid distractors for the treatment of syndromic craniosynostosis. A ten-year experience in a single center. Child's Nerv Syst 2014; 30: 1941.

47. Arangio P, Manganaro L, Pacifici A, Basile E, Cascone P. Importance of fetal MRI in evaluation of craniofacial deformities. $J$ Craniofac Surg 2013; 24: 773-6. doi: https://doi.org/10.1097/SCS. 0b013e318286988c

48. Al Kaissi A, Ben Chehida F, Kenis V, Ganger R, Radler C, Hofstaetter JG, et al. Broad spectrum of skeletal malformation complex in patients with cleidocranial dysplasia syndrome: radiographic and tomographic study. Clin Med Insights Arthritis Musculoskelet Disord 2013; 6: CMAMD.S11933. doi: https://doi. org/10.4137/CMAMD.S11933

49. Chan FC, Kawamoto HK, Federico C, Bradley JP. Soft-tissue volumetric changes following monobloc distraction procedure: analysis using digital three-dimensional photogrammetry system (3dMD). J Craniofac Surg 2013; 24: 416-20. doi: https://doi.org/ 10.1097/SCS.0b013e31827ff296

50. Wang H, Yuan J, Zhang Y, Qi Z, Wei M. Modified nasomaxillary and hard palatine osteotomy combined nasal implantation to correct binder syndrome. J Craniofac Surg 2013; 24: 200-3. doi: https://doi.org/10.1097/SCS.0b013e3182668752

51. Birgfeld CB, Saltzman BS, Luquetti DV, Latham K, Starr JR, Heike CL. Comparison of two-dimensional and three-dimensional images for phenotypic assessment of craniofacial microsomia. Cleft Palate Craniofac J 2013; 50: 305-14. doi: https://doi. org/10.1597/11-173

52. Lee DW, Ham KW, Kwon SM, Lew DH, Cho EJ. Dual midfacial distraction osteogenesis for crouzon syndrome: long-term follow-up study for relapse and growth. J Oral Maxillofac Surg 2012; 70: e242-e251. doi: https://doi.org/10.1016/j.joms.2011.11. 010

53. Manganaro L, Tomei A, Fierro F, Di Maurizio M, Sollazzo P, Sergi ME, et al. Fetal MRI as a complement to US in the evaluation of cleft lip and palate. Radiol Med 2011; 116: 1134-48. doi: https://doi.org/10.1007/s11547-011-0683-8

54. Miloro M. Mandibular distraction osteogenesis for pediatric airway management. J Oral Maxillofac Surg 2010; 68: 1512-23. doi: https://doi.org/10.1016/j.joms.2009.09.099

55. Bannink N, Nout E, Wolvius EB, Hoeve HL, Joosten KF, Mathijssen IM. Obstructive sleep apnea in children with syndromic craniosynostosis: long-term respiratory outcome of midface 
advancement. Int J Oral Maxillofac Surg 2010; 39: 115-21. doi: https://doi.org/10.1016/j.ijom.2009.11.021

56. Kaban LB, Seldin EB, Kikinis R, Yeshwant K, Padwa BL, Troulis MJ. Clinical application of curvilinear distraction osteogenesis for correction of mandibular deformities. J Oral Maxillofac Surg 2009; 67: 996-1008. doi: https://doi.org/10.1016/j.joms. 2009.01.010

57. Oyama A, Arnaud E, Marchac D, Renier D. Reossification of cranium and zygomatic arch after monobloc frontofacial distraction advancement for syndromic craniosynostosis. J Craniofac Surg 2009; 20(Suppl 2): 1905-9. doi: https://doi.org/10.1097/SCS. 0b013e3181b6c634

58. Eley KA, Witherow H, Hayward R, Evans R, Young K, Clark A, et al. The evaluation of bony union after frontofacial distraction. J Craniofac Surg 2009; 20: 275-8. doi: https://doi.org/10.1097/ SCS.0b013e31819921b2

59. Ponniah AJ, Witherow H, Richards R, Evans R, Hayward R, Dunaway D. Three-dimensional image analysis of facial skeletal changes after monobloc and bipartition distraction. Plast Reconstr Surg 2008; 122: 225-31. doi: https://doi.org/10.1097/ PRS.0b013e3181774308

60. Albuquerque MA, Cavalcanti MG. Computed tomography assessment of Apert syndrome. Braz Oral Res 2004; 18: 35-9. doi: https://doi.org/10.1590/S1806-83242004000100007

61. Santos DT, Miyazaki O, Cavalcanti MG. Clinical-embryological and radiological correlations of oculo-auriculo-vertebral spectrum using 3D-CT. Dentomaxillofac Radiol 2003; 32: 8-14. doi: https://doi.org/10.1259/dmfr/36409607

62. Reinhart E, Eulert S, Bill J, Würzler K, Phan The L, Reuther J. Typische merkmale des kraniofazialen wachstums beim FGFR3-assoziierten koronarnahtsynostosesyndrom (so genannte Muenke-Kraniosynostose). Mund-, Kiefer- und Gesichtschirurgie 2003; 7: 132-7. doi: https://doi.org/10.1007/s10006-002-0447-7
63. Roth DA, Gosain AK, McCarthy JG, Stracher MA, Lefton DR, Grayson BH. A CT scan technique for quantitative volumetric assessment of the mandible after distraction osteogenesis. Plast Reconstr Surg 1997; 99: 1237-47. doi: https://doi.org/10.1097/ 00006534-199705000-00005

64. Marsh JL, Celin SE, Vannier MW, Gado M. The skeletal anatomy of mandibulofacial dysostosis (Treacher Collins syndrome). Plast Reconstr Surg 1986; 78: 460-8. doi: https://doi.org/10.1097/ 00006534-198610000-00003

65. Slim K, Nini E, Forestier D, Kwiatkowski F, Panis Y, Chipponi J. Methodological index for non-randomized studies (minors): development and validation of a new instrument. $A N Z$ J Surg 2003; 73: 712-6. doi: https://doi.org/10.1046/j.1445-2197. 2003.02748.x

66. Li G. Patient radiation dose and protection from cone-beam computed tomography. Imaging Sci Dent 2013; 43: 63-9. doi: https://doi.org/10.5624/isd.2013.43.2.63

67. Tucunduva RM, Imada TS, Lopes IA, Rubira-Bullen IR, de Carvalho IM. Evaluation of interforaminal mandible region of individuals with pierre robin sequence and treacher collins syndrome through the cone-beam computed tomography. J Craniofac Surg 2016; 27: 219-21. doi: https://doi.org/10.1097/SCS. 0000000000002170

68. van Loon B, Maal TJ, Plooij JM, Ingels KJ, Borstlap WA, Kuijpers-Jagtman AM, et al. 3D Stereophotogrammetric assessment of pre- and postoperative volumetric changes in the cleft lip and palate nose. Int J Oral Maxillofac Surg 2010; 39: 534-40. doi: https://doi.org/10.1016/j.ijom.2010.03.022

69. Shaw WC, Semb G, Nelson P, Brattström V, Mølsted K, Prahl-Andersen B, et al. The Eurocleft project 1996-2000: overview. J Craniomaxillofac Surg 2001; 29: 131-40. doi: https://doi. org/10.1054/jcms.2001.0217 